\section{Rituelle Kommunikation}

\subsection{Definition des Konzepts}

Die Sprachwissenschaft hat den Begriff und das Konzept >Rituelle Kommunikation` von der vergleichenden Verhaltensforschung übernommen. Humanethologen unterscheiden eine Reihe von sogenannten >Ausdrucksbewegungen`, die in der Mimik, der Gestik, der Personaldistanz (Proxemik) und der Körperhaltung (Kinesik) zum Ausdruck kommen. Viele dieser Ausdrucksbewegungen haben sich zu spezifischen Signalen entwickelt. Ethologen definieren Ritualisierung als Veränderung von Verhaltensweisen im Dienst der Signalbildung. Die zu Signalen ritualisierten Verhaltensweisen sind Rituale. Im Prinzip kann jede Verhaltensweise zu einem Signal werden, entweder im Laufe der Evolution oder durch Konventionen, die in einer bestimmten Gemeinschaft gültig sind, die solche Signale kulturell entwickelt hat und die von ihren Mitgliedern tradiert und gelernt werden.

Voraussetzung für die Entwicklung einer Ausdrucksbewegung zu einem Signal ist, dass sie immer mit einem bestimmten Erregungszustand einhergeht - einem psychologischen und physiologischen $\mathrm{Zu}-$ stand höchster Aufmerksamkeit und Reaktionsbereitschaft eines Individuums auf wahrgenommene Eindrücke. Dieser Zustand lässt andere schnell erkennen, wie dieses Individuum auf das Wahrgenommene emotional reagiert, wie es disponiert ist und vor allem, was es nun zu tun beabsichtigt. Die Signale können sich in Form von physischen Phänomenen wie z. B. in Form von Erröten aber auch als Verhaltensweisen mit bestimmten Funktionen wie z. B. als freundliches bandstiftendes Verhalten oder als aggressives Verhalten ausdrücken - und sie werden von anderen auch sofort als solche Verhaltensweisen erkannt.

Bei der Ritualisierung zu Signalen werden Verhaltensweisen stärker ausgeprägt und eindeutiger gemacht, um ihre kommunikative Funktion zu verbessern. Die Bewegungen werden dabei vereinfacht, rhythmisch wiederholt und oft auch übertrieben ausgeführt; außerdem variieren sie in Hinsicht auf ihre Intensität. Dadurch wird das Verhalten von miteinander Interagierenden vorhersagbar - zumindest bis zu einem gewissen Grad - und mit dieser Zunahme von Vorhersagbarkeit des Verhaltens schaffen Rituale Sicherheit und Ordnung in der zwischenmenschlichen Interaktion (vgl. Eibl-Eibesfeldt 1984: 552 ff.; Huxley 1966; Senft 2014: 80 ff.). Dieses Schaffen und das Stabilisieren von sozialen Beziehungen ist eine der be- deutendsten Funktionen von Ritualen. Soziale Riten, die der Bandstiftung und der Aggressionsabblockung dienen, sind von größter Bedeutung für die Interaktion aller Lebewesen. Wir Menschen verfügen aber nicht nur über ritualisierte Formen nonverbalen Verhaltens, wir haben auch und vor allem ritualisierte Formen unseres Sprachverhaltens entwickelt. Der Begriff > Rituelle Kommunikation « subsumiert beide Verhaltensmuster.

In ihrer Anthologie "Ritual Communication« definieren Ellen Basso und Gunter Senft (2009: 1) das Konzept als:

"[...] an undertaking or enterprise involving a making of cultural knowledge within locally variant practices of speech-centered human interaction [...].

[...] [R]itual communication is artful, performed semiosis, predominantly but not only involving speech, that is formulaic and repetitive and therefore anticipated within particular contexts of social interaction. Ritual communication thus has anticipated (but not always achieved) consequences. As performance, it is subject to evaluation by participants according to standards defined in part by language ideologies, local aesthetics, contexts of use, and, especially, relations of power among participants."

Ausgehend von dieser Definition und im Rückgriff auf Senft $(1987,2009)$ wird im Folgenden dieses multimodale Konzept weiter ausgeführt und dann mit Beispielen von Formen ritueller Kommunikation der Trobriand-Insulaner von Papua-Neuguinea illustriert.

Sprecher natürlicher Sprachen müssen die Regeln des kommunikativen Verhaltens lernen, die in ihrer Sprachgemeinschaft gelten, sie müssen die Konstruktion der der Sprachgemeinschaft gemeinsamen Wirklichkeit verstehen und für sich selbst nachvollziehen. Verhaltensmuster und Verhaltensweisen müssen ebenfalls koordiniert und harmonisiert werden. Die so im Lernprozess nachvollzogene Konstruktion der gemeinsamen Wirklichkeit bedarf nun einer Absicherung in möglichen Bruchzonen des sozialen Miteinanders wie z. B. Konflikt, Kooperation und Konkurrenz. Diese Absicherung wird zu einem großen Teil durch Ritualisierung verbaler und nonverbaler Kommunikation gewährleistet, die kritische Situationen im Umgang miteinander entlastet und soziale Unstimmigkeiten reguliert, indem sie die harmonisierende Wirkung von Reden verstärkt, soziale Beziehungen schafft und stabilisiert und Emotionen, Antriebe und Absichten auf Distanz bringt. Damit wird 
Verhalten vorhersagbar gemacht und es werden wenn nötig - Spielräume eröffnet, in denen Handeln frei von Furcht vor möglichen Sanktionen erprobt werden kann.

Damit ist rituelle Kommunikation definiert als eine Form strategischen Handelns, die die Vorhersagbarkeit von Verhalten verstärkt, der Bindung und damit auch als Aggressionsblock dient und durch das ZurSprache-Bringen und Benennen der Gefahr einer Belastung des sozialen Miteinanders diese Gefahr selbst im sprachlichen Bereich bannen kann. Allerdings gelingt das nicht immer. Die Konstruktion der sozialen Wirklichkeit der Gemeinschaft muss nicht immer mit der Wahrnehmung einer bestimmten Situation durch ein Individuum übereinstimmen. Aggression, die dadurch entstehen kann, wird gewöhnlich durch die starke soziale Regel 'Sei nett!‘ unterdrückt, selbst wenn das Individuum das gar nicht sein will. Von daher können Emotionen gezügelt und verbale Ausbrüche unterdrückt werden. Besonders Gesellschaften, die ihren Mitgliedern nur wenig geschlossene Räume für ihre Privatsphäre bieten - wie z. B. die der Trobriander, deren Häuser aus Buschmaterialien mit > Wänden aus Palmblättern bestehen - müssen auf ein ausgeprägtes Taktgefühl ihrer Mitmenschen vertrauen. Manchmal muss man so tun, als ob man nichts von dem (mit-)gehört hat, was in einem Haus gesagt wird, und man muss schon früh lernen, dass man darüber auch nicht spricht. Die allgemeine Forderung von Taktgefühl, die Notwendigkeit, >nett $<$ zu sein und die positiven und erfolgreichen Effekte ritueller Kommunikation schaffen die notwendige soziale Harmonie auch in solchen Gesellschaften.

\subsection{Formen ritueller Kommunikation bei den Trobriandern von Papua- Neuguinea}

Im Folgenden werden nun verschiedene Formen ritueller Kommunikation am Beispiel der Trobriander angeführt, aber dazu bedarf es zunächst einer kurzen Vorstellung dieser Ethnie und ihrer Sprache. Die Trobriand-Insulaner gehören zur ethnischen Gruppe der Nord Massim. Die Inselgruppe, die sie bewohnen, liegt in der Mile Bay Provinz von Papua-Neuguinea. Sie sind brandrodende Gartenbauer, die vor allem Yams anpflanzen, exzellente Konstrukteure von Segelbooten, hervorragende Navigatoren und kunstvolle Schnitzer. Ihre Gesellschaft ist matrilinear mit patrilokaler Residenz. Die Sprache der Trobriander ist das
Kilivila, eine austronesische Sprache, die als westmelanesisch-ozeanische Sprache im $>$ Papuan tip cluster klassifiziert wird (vgl. Senft 1986: 6). Sie wurden vor allem durch die völkerkundlichen Arbeiten von Bronislaw Malinowski zu Beginn des 20. Jahrhunderts bekannt (vgl. Malinowski 1978).

Bei den Trobriandern - wie auch bei allen anderen Ethnien - können Formen ritueller Kommunikation im Hinblick auf ihre Vielfalt und Variation und der soziokulturellen Kontexte, in denen sie auftreten, äußerst komplex und situations-spezifisch oder relativ einfach und alltäglich sein - John Haviland (2009) spricht in seinen Untersuchungen zum zinakantekischen Tzotzil in Mexico von slittle rituals‘. Man kann diese verschiedenen Formen ritueller Kommunikation als ein Kontinuum oder eine Skala von zunehmender struktureller, kontextueller und soziokultureller Komplexität auffassen. Hier werden zunächst einfache und alltägliche, dann besondere und schließlich außergewöhnliche Formen ritueller Kommunikation vorgestellt.

\section{Einfache Formen ritueller Kommunikation}

$\mathrm{Zu}$ den wohl am meisten beschriebenen Ritualen des Alltags gehört das Grüßen. Obwohl die Trobriander von den Australiern europäische Grußformeln wie z. B. Guten Tag - Bwena kaukwa - übernommen haben, so besteht die informelle und gebräuchlichste Art des Grüßens in der Frage Ambeya? oder Ambe? - (Woher und) Wohin (des Wegs)?, die jeweils gegenseitig gestellt und genau und vollkommen ehrlich beantwortet wird. Dieses Begrüßungsritual versichert die Trobriander immer wieder aufs Neue ihres Eingebundenseins in ein sich um das Wohlergehen seiner Mitglieder sorgendes Gemeinwesen und damit auch ihres Schutzes durch das soziale Netz dieser Gemeinschaft, eines Schutzes, der ihnen auch einen sicheren Weg auf ihrer Insel und damit sichere Ankunft und Heimkehr gewährleistet. Sollte den Trobriandern auf ihren Wegen etwas zustoßen, dann können sie sicher sein, dass bekannt ist, wo man nach ihnen suchen muss, um ihnen - wenn nötig - Beistand und Hilfe zu leisten. Dieser Gruß ist damit nicht nur ein Ritual der freundlichen Begegnung, sondern er vermittelt als bindendes Ritual gleichzeitig Sicherheit im sozialen Netz der Gemeinschaft (vgl. Senft 2014: 1 f.).

$\mathrm{Zu}$ den Alltagsritualen gehören auf den TrobriandInseln - wie anderswo - auch verschiedene Formen von Bitten, Geben und Nehmen. Es ist ein Zeichen guten Benehmens, wenn man freigiebig Dinge wie z. B. Tabak, Zigaretten, Betelnüsse und Kokosnüsse mit an- 
deren teilt, sobald man darum gebeten wird. Der Druck, großzügig zu geben, ist bei dieser sozialimmanenten Form von Etikette so stark, dass er alle Rangunterschiede der trobriandischen Clanhierarchie verwischt. Wenn man diese Bitten missachtet, gilt man als krank oder als grob unfreundlich und wird mit dem von allen gefürchteten Schimpfwort tomekita Geizhals - sanktioniert. Wenn man allerdings zu häufig um etwas bittet, dann gilt man als tonigada - als Schnorrer. Am Beispiel der Art und Weise, wie Trobriander um Tabak bitten, werden hier nun alltägliche Formen von Bitten illustriert (vgl. Senft 2010: $271 \mathrm{ff}$.).

Manchmal, allerdings eher selten, wird ein Mittelsmann von jemandem gebeten, für ihn oder sie etwas zu erbitten. Dann fragt dieser Mittelsmann denjenigen, der im Besitz von Tabak ist:

\section{(1) Mtona/Minana magila Dieser Mann/Diese tobaki. Frau möchte Tabak.}

Die meisten Bitten sind aber sehr viel direkter, wie z. B. die Folgenden:

(2) Magigu babwayui!

(3) Agu tobaki!

Ich möchte rauchen! Mein Tabak (den ich rauchen will)!

(4) Ula tobaki!

(4) Mesta tobaki! Mein Tabak (den ich jemandem weitergeben werde)!

Gib mir etwas Tabak!

Die so Gefragten geben dann entweder kommentarlos etwas von ihrem Tabak ab, oder sie sagen:
(6a) M tobaki.
Dein Tabak
oder:
(6b) Kubwayui!
Rauche!

Normalerweise wird die Gabe ebenfalls kommentarlos genommen. Seltener bedankt man sich und sagt:
(7a) Agutoki!
Danke! oder:
(7b) Sena bwena.
Sehr gut.

Diese sozialimmanente Form von Etikette, großzügig zu geben, kann man als Norm verstehen, die das soziale Verhalten der Mitglieder einer Gruppe regelt. Solche Normen ordnen den Alltag eines Gemeinwesens und vermitteln damit den Mitgliedern dieser Gemeinschaft ein gewisses Maß an Sicherheit, weil sie das Verhalten der Gruppenmitglieder voraussagbar macht und damit auch die Einheit der Gruppe demonstriert. Von daher können diese Formen des Bittens um etwas als eine Kommunikationsform gesehen werden, die die sozial regulativen Verhaltensnormen immer wieder austestet, ihre Wirksamkeit demonstriert und damit zur Erhaltung der Gruppenharmonie und Gruppenbindung beiträgt. Von dieser normenkontrollierenden Funktion her ist die Direktheit der Kommunikation hier als rituelle Kommunikation konventionalisiert. Dass Verstöße gegen diese soziale Norm sanktioniert werden, ist als Strategie zur Erhaltung der gültigen Gruppennorm geradezu zwangsläufig.

\section{Besondere Formen ritueller Kommunikation}

$\mathrm{Zu}$ den besonderen Formen ritueller Kommunikation gehören anzügliche Verse und Lieder, die Malinowski (1978: 299) »ditties« genannt hat und die im Kilivila als > Vinavina $<$ bezeichnet werden (vgl. Senft 2010: $237 \mathrm{ff}$.). Viele dieser >Liedchen begleiten Spiele, die von Kindern und auch Erwachsenen gespielt werden. Die meisten von ihnen sind nicht nur anzüglich, sondern derb sexuell und oft auch pornographisch.

Jede Gesellschaft hat Bereiche, die als tabuisiert gelten und es gibt Dinge, über die man nicht spricht. Viele dieser Tabus betreffen die Sexualität und das Reden über sexuelle Dinge. Sexualität war bei den Trobriandern schon immer stark mit Tabus verbunden, auch wenn heute noch gilt, »that sex as such is not tabooed « (Malinowski 1987: 381). Malinowski listet eine ganze Reihe solcher Tabus auf und geht auch ausführlich auf Normen ein, die "Modesty in Speech and Behaviour « betreffen (Malinowski 1987: 402 ff.). Dass Tabus gebrochen werden - und zwar mit umso größerer Wahrscheinlichkeit, je strikter ihre Einhaltung gefordert wird - ist allgemein bekannt. Aber eine Gesellschaft kann sich der Einhaltung ihrer Tabus in den Bereichen, die ihr besonders wichtig sind, mit größerer Erfolgswahrscheinlichkeit dadurch versichern, dass sie ihren Mitgliedern zugesteht, in bestimmten Bereichen diese Tabus und ihre Verletzung zu thematisieren und sie sich sogar - in fiktiver Form, versteht sich - vorzustellen. Und genau deshalb sind Ventilsitten entstanden (vgl. Heymer 1977: 187). Zu den Ventilsitten im sprachlichen Bereich zählen vor allem Witze (vgl. Senft 2010: 152 f.). Im Folgenden wird dieses Phänomen am Beispiel von zwei der oben erwähnten Verse verdeutlicht. Der erste Vers begleitet ein Kreiselspiel (vgl. Senft 2010: 241), der zweite ein Fadenspiel (vgl. Senft/Senft 1986: 154 ff.). 
(8) Numwaye tomwaye, kusisusi va bweme idoketasi popu.

Alte Frau, alter Mann, ihr sitzt am Yamshaus sie ficken Scheiße.

In diesem Kinderreim wird die sexuelle Variante des Analverkehrs, die bei den Trobriandern als sexuelle Abirrung verpönt ist (vgl. Malinowski 1987: 382, 395 ff.), thematisiert. Solche Reime im Kindermund die übrigens auch in Deutschland z. B. von Peter Rühmkorf (1967) dokumentiert sind - überraschen weniger, wenn man vorher beobachtet hat, wie eine Großmutter mit ihren Enkelkindern ein Fadenspiel entwickelt hat, zu dem sie dann das folgende Verschen rezitiert:
(9) Tobabane, Tobabane, kwakeye lumta!

Kwalimati. Kusivilaga, kuyomama.
Tobabana, Tobabana, Du fickst Deine Schwester!

Du fickst sie zu Tode. Du drehst Dich um, Du bist matt und müde.
Dieser Vers thematisiert den Inzest zwischen Bruder und Schwester - den extremsten Fall des Verstoßes gegen das ausgesprochen strikte Bruder-Schwester-Tabu. Dieses "supreme taboo of the Trobriander « (Malinowski 1987: 437) besagt, dass Geschwister nichts von gegenseitigen erotischen Affären oder Bindungen wissen dürfen - zumindest nicht offiziell (vgl. Malinowski 1987: 433 ff., 451).

Wenn man Trobriander auf diese Tabubrüche, die in den Vinavina thematisiert sind, anspricht, dann rechtfertigt selbst ein ganz strikt an der gültigen Etikette orientierter Trobriander nicht nur die Tolerierung von, sondern auch die Freude an solchen Texten einfach mit dem Hinweis, dass das ja alles nur sopa ist.

Die Trobriander unterscheiden metasprachlich acht situations-intentionale Varietäten, die von bestimmten und ebenfalls metasprachlich benannten Genres konstituiert werden (vgl. Senft 2010). Eine dieser Varietäten heißt 〉biga sopa< - die Sprache des Witzes oder der Lüge, die indirekte Sprache, die Sprache, für die man nicht einsteht. Diese Varietät ist charakteristisch für trobriandische Rede - sie gilt als Standardregister trobriandischen Diskurses. Sie basiert auf der Tatsache, dass Kilivila - wie andere Sprachen auch Merkmale aufweist, die auch Vagheit und Ambiguität mit einschließen. Diese beiden Merkmale werden von den Sprechern des Kilivila als stilistische Mittel genutzt, um mögliche Konfrontationen oder für Trobriander zu große und zu aggressive Direktheit in be- stimmten Sprechsituationen zu vermeiden. Wenn Hörer signalisieren, dass sie einen bestimmten Sprechakt als beleidigend empfinden, dann können sich Sprecher immer von dem, was sie gesagt haben, distanzieren, indem sie es als sopa bezeichnen, als etwas, was sie ja gar nicht (ernst) gemeint haben. Sopa signalisiert, dass der Sprecher sich nicht der Wahrheit verpflichtet fühlt. Trobriandische Etikette verlangt dann von den Hörern, sich von den Äußerungen, die explizit als sopa deklariert wurden, nicht beleidigt zu fühlen. Tun sie es dennoch und zeigen das auch öffentlich, dann verlieren sie ihr Gesicht (vgl. Goffman 1967).

Die biga sopa wird in alltäglicher Konversation, im Small Talk, beim Flirten, in öffentlichen Debatten, in Mahnreden, in Liedern und Geschichten als rhetorisches Mittel verwendet, um mögliche Konflikte zu vermeiden und die Atmosphäre der Gesprächssituation zu entspannen. Die biga-sopa-Varietät erlaubt es auch, kritische Argumente vorbringen zu können, weil sie es den Sprechern ermöglicht, ihre wahren Gedanken $\mathrm{zu}$ verschleiern und verbal auf spielerische Weise mit etwas nicht einverstanden zu sein, ohne Gefahr zu laufen, sich persönlich allzu sehr zu exponieren. Außerdem kann die biga-sopa-Varietät auch dazu benutzt werden, um jemanden zu verspotten. Als Mittel der Ironie und Parodie kann sie auch abweichendes Verhalten kritisieren und so auch korrigieren - ohne Furcht vor möglichen negativen Konsequenzen dieser Kritik. Und schließlich gewährt die biga-sopa-Varietät nicht nur Erwachsenen, sondern auch Kindern die Lizenz zum verbalen Tabubruch und ermöglicht damit den Gebrauch der sogenannten >biga-gaga -Varietät der verpönten schlechten, unangemessenen Sprache mit fast all ihren Beleidigungen (außer den schlimmsten), Kraftausdrücken und Flüchen.

Genres der biga sopa, die eindeutig Merkmale der biga gaga aufweisen - wie Witze und Lügen (sopa), Geschichten (kukwanebu), Tratsch (kasilam) und Lieder (wosi), Liedchen (Vinavina) und Ernterufe (sawila) werden ausschließlich als sopa - klassifiziert, als Spielerisches und damit als etwas für die trobriandische Gesellschaft nicht Reales sondern Fiktives. Damit stellt die biga sopa ein Forum zur Verfügung, wo das Brechen von Tabus - und damit auch der Gebrauch der sschlechten Sprache - erlaubt ist, solange das eben nur verbal geschieht! Dieses Forum erlaubt eine besonders markierte Form der Kommunikation über etwas, über das man sonst eigentlich nicht spricht. Auf den Trobriands ist es also eine Sache, Normen und Tabus in der Realität zu verletzten, aber eine andere, das auf fiktive - rein im Verbalen verhaftete - Weise zu tun. 
Fassen wir zusammen: die biga-sopa-Varietät kanalisiert Emotionen, dient der Aggressionskontrolle und hält alle Möglichkeiten zum freundlichen Sozialkontakt offen. Dieses Konzept mit seinen Spannung vermeidenden Funktionen sichert die Harmonie innerhalb der trobriandischen Gesellschaft und trägt dazu bei, die soziale Konstruktion der Realität der Trobriander aufrechtzuerhalten. Die biga sopa liefert einen wichtigen Rahmen und ist gleichzeitig ein effektives und dynamisches Werkzeug für rituelle Kommunikation, eine Form der Kommunikation, die ausgesprochen charakteristisch und äußerst bedeutsam für die soziale Interaktion der Trobriander ist.

\section{Außergewöhnliche Formen der rituellen Kom- munikation}

Jeder Tod bedeutet einen Verlust für die Hinterbliebenen. Je kleiner die Gruppe ist, die davon betroffen wird, desto größer ist dieser Verlust, weil er das Verhältnis der Gruppenmitglieder in ihrem sozialen Netzwerk stört und möglicherweise auch gefährdet. In dieser Situation kommt es leicht zu Konflikten. Der Verlust eines Menschen impliziert Frustrationen, die nicht nur zu Trauer führen, sondern auch leicht in Ärger, Wut und Aggression umschlagen können. Diese Gefühle müssen kanalisiert werden, um größeren Schaden zu verhindern. Das ist wohl einer der Gründe, warum Kulturen Trauerrituale entwickelt haben. Wenn wir unter >Ritual` auch Ausprägungen eines »distanced reenactment of situations of emotional distress « (Scheff 1977: 488) verstehen, dann sind Trauerzeremonien ritualisierte Handlungen par excellence.

Die Trauerzeremonien auf Trobriand sind geprägt von öffentlichen Totenklagen und Ausbrüchen hemmungslosen Weinens (vgl. Senft 1985). Diese öffentliche Trauer in all ihren verbalen und nonverbalen Formen und ihren verschiedenen Graden an Komplexität drückt nicht nur Kummer und Gram aus, sondern kanalisiert auch Emotionen, besonders Aggressionen, und trägt damit entscheidend zur Bandstiftung in der Gruppe bei, die vom Verlust eines ihrer Mitglieder betroffen ist. Die Trauernden werden nicht allein gelassen, Trauer wird zu einem gemeinsamen Erlebnis und dadurch wird die Gefahr, dass das soziale Netz der Gruppe zerstört wird, gebannt. Dieses Weinen und Klagen um Verstorbene ist in der Tat hoch ritualisiert und eine wichtige Form der rituellen Kommunikation auf den Trobriand-Inseln.

Bei diesen Trauerklagen wird - offenbar zur Erleichterung der Überwindung des Verlusts - der reli- giös-weltanschauliche Überbau der Trobriander eingebunden. Das geschieht dadurch, dass vor dem aufgebahrten Toten eine Gruppe älterer Frauen Lieder singt, die sonst nur im Rahmen der Erntefeste gesungen werden. Diese wosi milamala mit ihren meist erotischen Texten beziehen sich auf >Personen`, Ereignisse und Orte im nach dem Glauben der Trobriander unterirdischen Totenreich der baloma, der Totengeister bei der Insel Tuma (vgl. Senft 2011). Sie beschreiben das dort unbeschwerte süße Leben der baloma. Diese milamala-Lieder sind Manifestationen des Glaubens der Trobriander an einen unsterblichen Geist (baloma). Diese Totengeister können wiedergeboren werden, sie können aber auch unsichtbar in ihre Dörfer, in denen sie als Menschen gelebt hatten, zurückkehren. Diese Glaubensvorstellung liefert das einzige Bindeglied zwischen Trauerfeier und Totenklage einerseits und Erntefest und Ernteliedern andererseits: Bei den Erntefesten besuchen nämlich nach dem Glauben der Trobriander die baloma in aller Regel auch wieder ihre Dörfer.

Vor diesem religiös-weltanschaulichen Hintergrund kann man die Funktion der wosi milamala als Bestandteil der Trauerklage folgendermaßen erklären: Die Lieder sollen mit ihrer Beschreibung des unbeschwerten Lebens der baloma den Totengeistern der Verstorbenen den Abschied aus der Gruppe ihrer Verwandten und Freunde erleichtern; sie sollen aber sicherlich auch die Hinterbliebenen trösten, ist der Tod der Betrauerten doch nur ein Passageritus von einer Existenzform in eine andere. Der rituell-kommunikative Verweis im Lied auf dieses religiös-weltanschauliche Überbauwissen als konstitutives Element der trobriandischen sozialen Wirklichkeit trägt mit dazu bei, die bei einem Trauerfall unausbleiblichen Emotionen zu kanalisieren und zu kontrollieren. Er trägt damit auch dazu bei, dass der Zusammenhalt der Gruppe gewahrt und ihre Existenz im sozialen Geflecht der Ethnie gesichert bleibt.

Welche Funktion kommt aber den wosi milamala im Zusammenhang mit ihrem primären Kontext nämlich den Erntefeiern - zu? Die Erntefeiern, die sogenannten milamala-Feste sind für die Trobriander die wichtigste Zeit im Verlauf eines Jahres. Sie beginnen nach der Yams-Ernte am Tag nach dem Einfüllen der Knollen in die zum Teil prächtig geschmückten Yams-Vorratshäuser. Vor Sonnenaufgang schmücken sich die Männer eines Dorfes und einige Frauen sehr sorgfältig und treffen sich dann auf dem Dorfplatz mit einer Gruppe von Sängern und Trommlern, die sich dort schon im kleinen Rund aufgestellt haben. Auf ein 
Zeichen des Tanzmeisters stimmen die Sänger dann Lieder an, die Trommler untermalen diese Lieder rhythmisch, und die Tänzer beginnen mit ihren Schreit- und Rundtänzen, die je nach den verschiedenen Liedern variieren.

Diese milamala-Lieder klingen, als kämen sie aus einer anderen Welt - und das tun sie eben im Glauben der Trobriander auch tatsächlich. Die Eröffnung des milamala-Fests kann über drei Stunden dauern - und die Festlichkeiten selbst können sich über mehr als einen Monat hinziehen. Nahezu jeden Tag werden vor allem abends und manchmal auch die ganze Nacht hindurch immer wieder die milamala-Lieder gesungen. In dieser Zeit erholen sich die Trobriander von den Mühen der äußerst anstrengenden Gartenarbeit, die in der Ernte ihren Höhepunkt gefunden hat. Die Zeit des milamala ist geprägt von allgemeiner Freude, Geselligkeit, gegenseitigen Besuchen von Dorfgemeinschaften, Tanz und amourösen Abenteuern der unverheirateten Jugendlichen. Die das milamalaFest einleitenden Tänze und Gesänge begrüßen dabei auch die Totengeister, die für diese Feierlichkeiten als unsichtbare Gäste in ihre Dörfer zurückkehren, um mit ihren Verwandten und Freunden gemeinsam diese Zeit ausgelassener Freuden zu feiern. Die milamala-Lieder werden nämlich in einer besonderen, archaischen Varietät des Kilivila - in der biga baloma der Sprache der Totengeister, die auch biga tommwaya - Sprache der Ahnen genannt wird, gesungen. Damit erweisen die Lebenden den Totengeistern ihre Ehre. Die milamala-Lieder bestehen aus einzelnen Strophen, die in einem mehr oder minder komplexen Liedzyklus das Leben der Totengeister in ihrem unterirdischen Totenreich bei der Insel Tuma beschreiben. Der Liedzyklus »Wosi Pilugwana«, der aus 6 Einzelgesängen besteht, illustriert diese milamala-Lieder (Senft 2011: 72 f.):

(10) Wosi Pilugwana

1 Vadenisa Bwiyariga yakayobu yamvedoku.

2 Kapisina Kwe'ineoli gidiviligu

Ne'oli.

3 Kumidorigu mweyuva - Mein scharfes Gewürz mamidorim mweyuva - wie gut das tut, scharfes Gewürz - vana-simgeori. und wohlriechend

4 Sogu Yaurivori ka'uvamapu maka'i

kakamapu mabwita.

$5 \quad$ Natokwabu ugwaraiKraut - ganz frisch. Geliebte Yaurivori wir treffen uns Worte der Liebe, wir tauschen die Blumen im Haar. Natokwabu - da geht sie, bleibt steh'n -

nukulibusa, Pilugwana. doch Du ziehst's hinaus auf die See, das Kanu Pilugwana.

6 Kemyovau, ugwenegu - Neu ist's Kanu, 's ist mein Kanu bakana'ira o papala. bald lieg' ich mit ihr auf hölzerner Plattform.

Die Mehrheit der Trobriander hat ihre Kompetenz zum Verständnis dieser Varietät verloren; trotzdem werden die Liedtexte immer noch in traditioneller unveränderter Form weitergegeben und so - obwohl unverständlich geworden - auch noch von jungen Leuten gesungen. Neben der zentralen Bedeutung der wosi milamala als ein die allgemeinen Feierlichkeiten sowohl abschließender als auch zum neuen Feiertag überleitender sozialer Ritus des gemeinsamen Singens ist dieser Faktor ein entscheidendes Indiz für die Wichtigkeit, die diesen Liedern offenbar zuerkannt wird.

In einer relativ lang andauernden Zeit ausgelassener Freude ist eine gelockerte Auslegung sozialer Regeln und Normen zu erwarten. Dass diese Lockerung der Sitten und Normen gerade in einer Zeit erhöhter Sensualität und Sensitivität nicht dazu führt, dass die Sozialgemeinschaft gefährdet wird, scheint in den milamala-Liedern begründet zu sein. Sie erinnern als eine außergewöhnliche Form ritueller Kommunikation an die auch für das Leben der baloma im Totenreich geltenden Normen des sozialen Miteinanders und an die wenn auch unsichtbare - Präsenz der Ahnen, die nicht durch unziemliches Verhalten beleidigt werden dürfen. Sie könnten dieses Verhalten dann sanktionieren, in dem sie z. B. den Ernteerfolg eines Trobrianders oder die Gebärfähigkeit einer Trobrianderin beeinflussen. Im Bewusstsein dieser Tatsache muss jeder sein Verhalten und Handeln ausrichten. Niemand würde es wagen, die baloma durch schlechtes Benehmen - und dazu gehört auch Eifersucht zwischen unverheirateten Jugendlichen - zu verärgern und zu beleidigen. Deshalb kann es auch nicht zu einer Gefährdung der Gemeinschaft während der Erntefeiern kommen. Die manchmal bis zum frühen Morgen gesungenen mila- 
mala-Lieder mahnen und erinnern immer wieder aufs Neue an die Präsenz der Totengeister. Sie versichern damit die Gemeinschaft eines gleichsam transzendenten Regulativs für das Verhalten ihrer Mitglieder.

Die wosi milamala werden also nicht nur zu außergewöhnlichen Anlässen gesungen, sondern sie sind selbst aufzufassen als eine außergewöhnliche Form ritueller Kommunikation, die mit ihren Funktionen der Bindung sowie der Normen- und Aggressionskontrolle die Konstruktion der sozialen Realität des Gemeinwesens sichert und darüber hinaus in eigener und einmaliger Form in diesem Gemeinwesen tradierte Kultur wahrt.

\subsection{Zusammenfassung}

Die hier beschriebenen Formen ritueller Kommunikation bei den Trobriandern tragen dazu bei, die Konstruktion der sozialen Wirklichkeit dieser Inselbewohner abzusichern. Grüße und Formen von Bitten und großzügigem Geben haben sozial-integrative Funktionen, die bandstiftend sind und das Verhältnis der Mitglieder einer Gemeinschaft untereinander stabilisieren. Die trobriandischen vinavina-Verse und -Liedchen, besonders die anzüglichen, stellen als konstituierende Elemente der biga sopa, der uneigentlichen Sprache (für die man nicht einsteht), ein Forum zur Verfügung, auf dem Dinge gesagt werden können, über die man sonst eigentlich nicht spricht. Damit tragen sie in einer Gemeinschaft, die das strikte Beachten bestimmter Tabus fordert, entscheidend zur Kontrolle von Emotionen, Frustrationen und Aggressionen bei. Und die Erntelieder als Manifestationen des trobriandischen Glaubens an unsterbliche Totengeister, die Beleidigungen durch ungebührliches Betragen sanktionieren können, tragen sowohl während der Erntefeiern als auch nach einem Todesfall ebenfalls entscheidend zur Bandstiftung und zur Emotionskontrolle bei.

Mit Ausnahme vielleicht der relativ einfachen Formen des Grüßens und des Bittens sind alle anderen hier diskutierten Formen ritueller Kommunikation bei den Trobriand-Insulanern Ergebnisse einer kunstvoll ausgeführten Semiose; sie sind formelhaft und repetitiv, werden deshalb auch in bestimmten Kontexten sozialer Interaktion antizipiert und haben in aller Regel auch vorhersehbare (wenn auch nicht immer eintreffende) Konsequenzen. Sie werden interaktiv realisiert und können darum auch von den miteinander Interagierenden beurteilt werden, und zwar aufgrund von Standards, die durch Sprachideologien,
Kontexte und Machtverhältnisse zwischen den Betroffenen vorgegeben sind. Darüber hinaus können sie auch noch wichtige Aspekte kultureller Identität einer bestimmten Ethnie bewahren. Damit werden sie voll und ganz der zu Anfang dieses Artikels von Basso und Senft (2009: 1) gegebenen Definition von ritueller Kommunikation gerecht.

\subsection{Eine mutige Hypothese für die weitere Forschung}

Der Humanethologe Irenäus Eibl-Eibesfeldt (1984: $610 \mathrm{ff}$.) geht davon aus, dass Rituale und Formen ritueller Kommunikation auf sogenannte elementare Interaktionsstrategien zurückgeführt werden können. Er argumentiert, dass alle Menschen über ein endliches Inventar solcher konventionalisierter Strategien verfügen und nimmt an, dass diese Strategien universal sind. Er unterscheidet dabei Strategien der Bandstiftung und Gruppeneinheit, Strategien des sozialen Lernens und Lehrens, des Rangstrebens und agonale Strategien, die er etwas martialisch als Strategien der Feindbekämpfung bezeichnet. Für ihn folgt die Art und Weise, wie Menschen in unterschiedlichen Kulturen versuchen, Ansehen und Status zu gewinnen, um etwas bitten, jemanden einladen oder Aggressionen vermeiden ein und denselben grundlegenden Mustern. Auf der Basis seiner Feldforschungen kommt er zu dem folgenden Schluss (Eibl-Eibesfeldt 1984: 645):

"Das äußerliche Kleid der menschlichen Umgangsformen variiert erheblich von Kultur zu Kultur. Bei genauerer Betrachtung lassen jedoch die Strategien sozialer Interaktion einen universalen Aufbau erkennen, der auf ein innen zugrundeliegendes universales Regelsystem verweist. Im Rahmen dieses Regelsystems können Verhaltensweisen verschiedenen Ursprungs, aber gleicher Funktion einander als funktionale Äquivalente ersetzen, auch Sätze können für Handlungen eintreten."

Im Verständnis des Humanethologen können also viele Rituale und Formen ritueller Kommunikation als Ausdifferenzierungen dieser elementaren Interaktionsstrategien verstanden und auf sie zurückgeführt werden. Trotz aller vielfältigen Unterschiede sind sie letztlich doch nur kulturspezifische Ausprägungen dieser Strategien. Das ist sicherlich eine gewagte, aber auch eine sehr interessante Hypothese, die weitere vielversprechende Ansätze zur Erforschung ritueller Kommunikation eröffnet. 


\section{Literatur}

Basso, Ellen B./Senft, Gunter (2009): Introduction. In: Gunter Senft/Ellen B. Basso (Hg.): Ritual Communication. Oxford, 1-19.

Eibl-Eibesfeldt, Irenäus (1984): Die Biologie des menschlichen Sozialverhaltens. Grundriß der Humanethologie. München/Zürich.

Goffman, Erving (1967): Interaction Ritual: Essays on Faceto-Face Behavior. New York.

Haviland, John (2009): Little rituals. In: Gunter Senft/Ellen B. Basso (Hg.): Ritual Communication. Oxford, 21-49.

Heymer, Armin (1977): Vocabulaire Éthologique: Allemand - Anglais - Français. Berlin.

Huxley, Julian (1966): A discussion on ritualization of behaviour in animals and man. Organized by Sir Julian Huxley, F. R. S. published by the Royal Society, Burlington. In: Philosophical Transactions of the Royal Society of London, Serie B, Biological Sciences No. 772 (Bd. 251), 247-526.

Malinowski, Bronislaw (1978): Argonauts of the Western Pacific. An Account of Native Enterprise and Adventure in the Archipelagoes of Melanesian New Guinea [1922]. London.

Malinowski, Bronislaw (1987): The Sexual Life of Savages in North-Western Melanesia [1929]. Boston.
Rühmkorf, Peter (1967): Über das Volksvermögen. Exkurse in den literarischen Untergrund. Reinbek bei Hamburg.

Scheff, Thomas J. (1977): The distancing of emotion in ritual. In: Current Anthropology 18, 483-505.

Senft, Gunter (1985): Trauer auf Trobriand - eine ethnologisch/linguistische Fallstudie. In: Anthropos 80, 471-492.

Senft, Gunter (1986): Kilivila - the Language of the Trobriand Islanders. Berlin/New York.

Senft, Gunter (1987): Rituelle Kommunikation auf den Trobriand-Inseln. In: Zeitschrift für Linguistik und Literaturwissenschaft 65, 105-130.

Senft, Gunter (2009): Trobriand islanders' forms of ritual communication. In: Gunter Senft/Ellen B. Basso (Hg.): Ritual Communication. Oxford, 81-101.

Senft, Gunter (2010): The Trobriand Islanders' Ways of Speaking. Berlin/New York.

Senft, Gunter (2011): The Tuma Underworld of Love - Erotic and other narrative songs of the Trobriand Islanders and their spirits of the dead. Amsterdam/Philadelphia.

Senft, Gunter (2014): Understanding Pragmatics. London/ New York.

Senft, Barbara/Senft, Gunter (1986): Ninikula - Fadenspiele auf den Trobriand-Inseln, Papua-Neuguinea. In: BaesslerArchiv, Beiträge zur Völkerkunde NF 34, 93-235.

Gunter Senft 